\title{
Gliomatosis Cerebri: Current Understanding and Controversies
}

\author{
Surabhi Ranjan ${ }^{1}$ and Katherine E. Warren ${ }^{2 *}$ \\ ${ }^{1}$ Neuro-Oncology Branch, National Cancer Institute, National Institutes of Health, Bethesda, MD, United States, \\ ${ }^{2}$ Pediatric-Oncology Branch, National Cancer Institute, National Institutes of Health, Bethesda, MD, United States
}

OPEN ACCESS

Edited by:

Rohan Ramakrishna, Weill Cornell Medical College, United States

Reviewed by: Kamalakannan Palanichamy, The Ohio State University Columbus, United States Luis Del Valle, LSU Health Sciences Center New Orleans, United States

${ }^{*}$ Correspondence: Katherine E. Warren warrenk@mail.nih.gov

Specialty section:

This article was submitted to Neuro-Oncology and

Neurosurgical Oncology, a section of the journal Frontiers in Oncology

Received: 27 April 2017 Accepted: 24 July 2017 Published: 07 August 2017

Citation:

Ranjan S and Warren KE (2017) Gliomatosis Cerebri: Current Understanding and Controversies. Front. Oncol. 7:165. doi: 10.3389/fonc.2017.00165
Gliomatosis cerebri (GC) is a rare, extensively infiltrating glioma involving multiple contiguous lobes of the brain. This lethal disease affects all age groups, and the majority of patients have a poor outcome despite aggressive treatment. Despite its initial recognition in 1938, GC remains a controversial entity with little consensus in its definition, histology, or treatment. The majority of GC tumors are astrocytic, although mixed phenotypes have been identified. Treatment of GC is challenging as surgery is generally not an option due to the extensive areas of brain involved, the benefit of radiation therapy is unclear, and no chemotherapy has proven efficacy. Due to the rarity of the disease and its heterogeneity, both at histopathological and molecular levels, it is difficult to conduct clinical trials tailored for this diagnosis. This review summarizes our current knowledge, examines clinical studies focusing on the treatment of GC, highlights ongoing challenges, and discusses the recent molecular insights into adult and pediatric GC. We conclude that, although no longer recognized as a distinct pathological entity, GC represents a unique disease phenotype. Given the histologic and molecular overlap with other diffuse gliomas, the research emphasis should be on investigating its unique invasive biology.

Keywords: gliomatosis cerebri, glioma, primary gliomatosis cerebri, secondary gliomatosis cerebri, review

\section{INTRODUCTION}

Gliomatosis cerebri (GC) is a rare, diffusely infiltrating glial brain tumor. Prognosis is poor, with $26-52 \%$ surviving less than a year from symptom onset $(1,2)$. GC is a controversial disease entity as no consensus exists regarding its definition, histopathology, and treatment. The 2007 World Health Organization (WHO) classification of central nervous system (CNS) tumors defined GC as an extensively infiltrative diffuse glioma involving at least three cerebral lobes; however, the revised 2016 WHO CNS classification no longer identifies GC as a distinct pathological entity (3). Rather, this new classification designates it as a special pattern of growth and categorizes it under the various subtypes of diffuse gliomas. While this approach is legitimate within the scope of a pure histopathological classification, it implies that GC is simply a grand manifestation of diffuse glioma. Patients with GC generally have a worse prognosis compared to patients with diffuse glioma of corresponding grades. It is unclear whether this is due to the distinct GC biology causing extensive invasiveness or merely because of the fact the due to the large areas of brain involved, treatment options are limited. While progress has been made in the biological understanding and treatment of various gliomas, little progress has been made in understanding GC. The rarity of the disease, lack of in-depth understanding of the tumor biology, variation across histopathological grading, variability in patient outcomes, and the lack of durable response to therapies are major obstacles toward establishing standard treatments. This review highlights our current understanding of GC and discusses recent molecular diagnostics which may help in tailoring more efficacious therapeutic regimens. The question as to whether GC is a distinct disease entity or a distinctive phenotype of diffuse glioma will require additional investigations. 
The histological, clinical, and radiographic classification of GC is not universally agreed upon. Classification systems are cumbersome and their clinical utility unclear. Conventionally, GC has been classified as primary or secondary GC $(4,5)$. Primary GC arises de novo and is further subclassified as type I (classic) when no obvious mass is present, or type II, where a diffuse infiltrative pattern coexists with an associated tumor mass (2). Secondary GC is defined as an infiltrative spread of tumor cells from a previously diagnosed glioma and is frequently associated with prior radiation or antiangiogenic therapy $(2,4)$. In light of the 2016 WHO classification update, GC is further subcategorized according to histopathologic grade and molecular findings, i.e., GC-diffuse astrocytoma, IDH-mutant; GC-diffuse astrocytoma, IDH-wildtype; GC-anaplastic astrocytoma, IDH-mutant; GC-anaplastic astrocytoma, IDH-wildtype; GC_-glioblastoma, IDH-mutant; GC-glioblastoma, IDH-wildtype; GC-oligodendroglioma, IDH-mutant and 1p/19q-codeleted; GC-anaplastic oligodendroglioma, and IDH-mutant and 1p/19q-codeleted.

\section{DIAGNOSIS}

Gliomatosis cerebri spans across all age groups but is more common in adults. The median age at diagnosis ranges from 46 to 53 years $(1,6,7)$ with a slight male predominance (sex ratio, 1.4 ) (6). Clinical presentation is variable and typically insidious, often delaying the diagnosis by months or years. Common presenting symptoms may be location dependent and include focal weakness, sensory loss, seizure, progressive headache or manifestations of increased intracranial pressure, memory deficit with "dementia-like" features, and other constitutional symptoms $(2,4,5,8)$. Common clinical signs include corticospinal tract, spinocerebellar, sensory-motor and visual field deficits, cranial neuropathies, papilledema, and myelopathy $(2,9)$. Children commonly present with seizures, developmental delay, increased intracranial pressure, and cognitive changes $(10,11)$. On examination, hemiparesis, ataxia, cranial neuropathies, altered mental status, tremor, and ataxia are observed (11). There are no classical symptoms or signs of GC owing to the extensive and unpredictable invasion of tumor cells into cerebral hemispheres and deep midline structures.

Before the magnetic resonance imaging (MRI) era, many patients with GC died without an established diagnosis and GC was determined at autopsy. Currently, GC is diagnosed radiographically by MRI along with histopathologic confirmation of an astrocytic process $(1,12,13)$. Brain MRI shows a T1-weighted hypo- or iso-intensity and T2-weighted or FLAIR hyperintensity in the involved areas (Figure 1). There may be diffuse infiltration of the cortex, poor gray-white matter delineation, enlargement of affected cerebral structures and thickened gyri $(14,15)$ Enhancement patterns are variable, with focal, multifocal, or
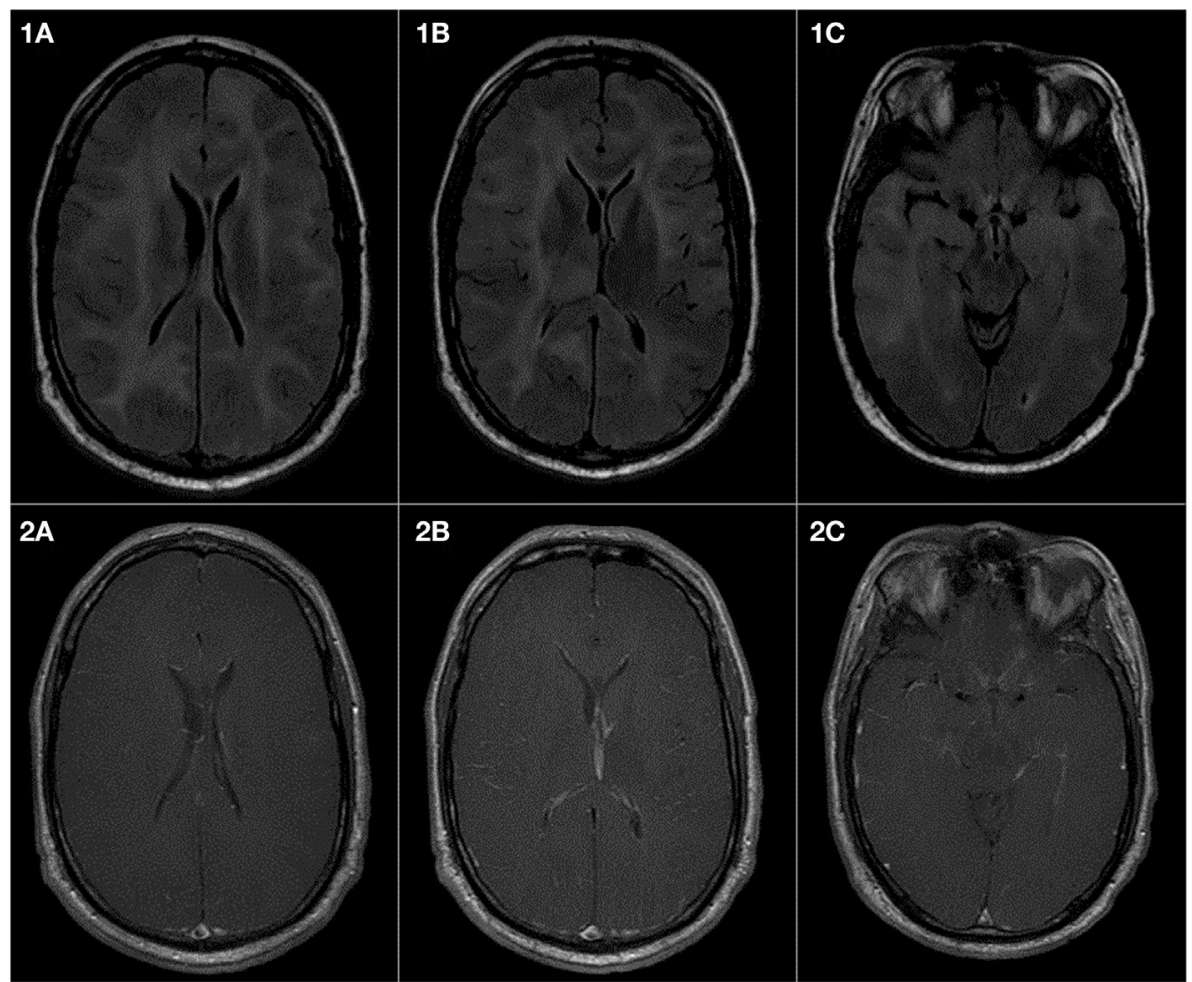

FIGURE 1 | Gliomatosis cerebri presenting in a 43-year-old man as a syncopal episode, insidious onset of slurred speech and left leg weakness. Pathology was consistent with IDH-wildtype anaplastic astrocytoma. (1A-1C) are FLAIR sequences demonstrating extensive abnormalities in the white matter of both cerebral hemispheres presenting as high signal intensity. There is also an involvement of midbrain and the right thalamus. The right temporal lobe shows more extensive abnormality as compared to the left temporal lobe. (2A-2C) are T1 sequences postcontrast and show no abnormal increased enhancement in the brain parenchyma or in the meninges. 
nodular gadolinium-enhancement in $16-56 \%$ patients $(1,5)$. Radiographic differential diagnoses include multiple sclerosis, progressive multifocal leukoencephalopathy, Behcet's disease, ischemia, viral encephalitis, vasculitis, subacute sclerosing panencephalitis, ischemia, and other CNS inflammatory diseases $(5,12)$. In children, GC can be misdiagnosed as encephalitis, acute disseminated meningoencephalitis, idiopathic intracranial hypertension, acute disseminated encephalomyelitis, tubercular encephalitis, leukodystrophy and primary progressive multiple sclerosis $(16,17)$.

On MR spectroscopy (MRS), the choline (Cho)/creatine (Cr) ratio is increased and the $\mathrm{N}$-acetylaspartate/Cr ratio is decreased, as observed in other malignant brain tumors $(15,19)$. MRS cannot reliably differentiate GC from encephalitis, demyelinating disease, progressive multifocal leukoencephalopathy, or hemorrhage $(20,21)$. Perfusion MR findings typically demonstrate lack of elevation of mean relative cerebral blood volume (22), corresponding to a relative lack of vascular angiogenesis. Fludeoxyglucose-positron emission tomography (FDG-PET) is not particularly useful for initial diagnosis as hypometabolism (23) or hypermetabolism (24) is seen in areas with infiltration; however, FDG-PET can be of value in following patients longitudinally for the extent of tumor involvement and treatment response assessment.

\section{HISTOPATHOLOGY AND MOLECULAR CLASSIFICATION}

Most GC tumors are astrocytic, although oligodendroglial and mixed phenotype can rarely be seen. The gross anatomy remains intact, but affected areas appear firm, edematous, with flattened gyri and loss of gray-white distinction $(25,26)$. Though histological grading encompasses gliomas from grades II through IV, the clinical behavior of the tumor is consistent with an aggressive malignancy. GC classically has a diffuse, irregular parenchymal infiltration of glial cells, in contrast to the destructive, necrotic pattern seen in high-grade gliomas. Histologic exam reveals small, astrocytic cells with elongated fusiform nuclei, readily identified by staining for glial fibrillary acidic protein (Figure 2) $(11,25)$. In contrast to high-grade gliomas, neovascularization, significant mitotic activity and necrosis are not common (11). Because most tissue is obtained from a small biopsy specimen, the degree of intratumoral heterogeneity is unknown.

Our clinical and genetic understanding of many brain tumors are now refined by genomic studies and epigenome-wide methylation profiling, which have unraveled molecular subgroups in tumors such as glioblastoma, medulloblastoma, and ependymoma (27-30). However, application of these studies has not been insightful for GC. Surprisingly, molecular and methylation profiling showed that in both adults and children, there are no characteristic histologic features or molecular subgroups exclusive to this diagnosis. In a study of 25 adults with GC, patients were found to have isocitrate dehydrogenase (IDH) mutant astrocytoma, IDH mutant and $1 \mathrm{p} / 19 \mathrm{q}$ codeleted oligodendroglioma or IDH wild type glioblastoma (RTK1, classic, mesenchymal, or H3F3AG34 mutant) (1). Likewise, when Broniscer et al. analyzed 32 pediatric and adolescent patients with types I and II GC, their

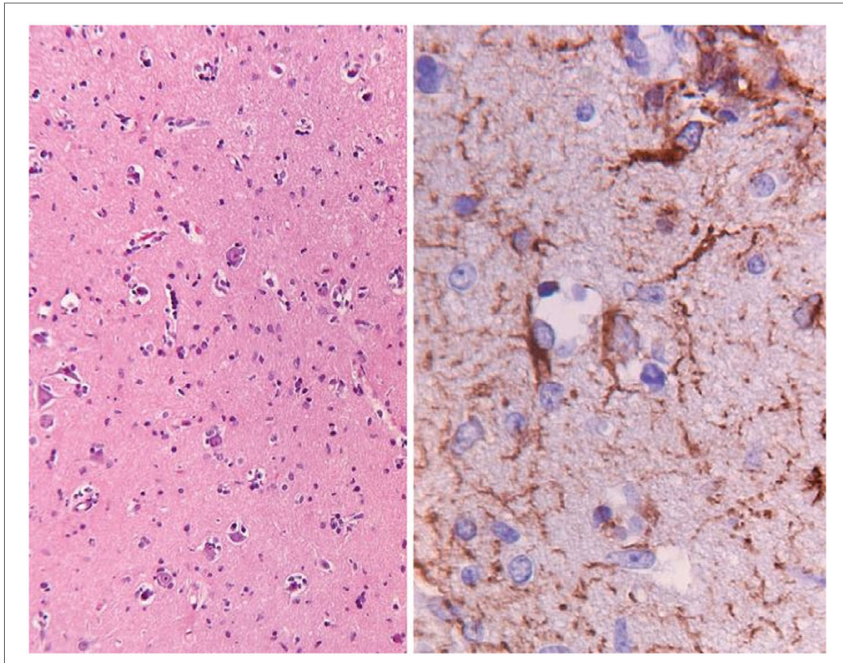

FIGURE 2 | This is a pathology slide of a World Health Organization (WHO) grade II astrocytoma manifesting as gliomatosis cerebri. The left-hand panel shows hematoxylin-eosin-stained section of cortical tissue with a slight increase in cellularity (objective $\times 2.5$ ). The right-hand panel shows GFAP staining for astrocytic cells (objective $\times 40$ ), demonstrating somewhat irregular, atypical positive-staining cells. Buis et al. (18) (C) The Author(s) 2011. Reproduced with permission of Springer.

DNA methylation profile corresponded with known pediatric glioma molecular subgroups, including IDH mutant (17\%), G34 (22\%), mesenchymal (17\%), and RTK I “PDGFRA” (44\%) (13). All tumors were astrocytic and no codeletion of $1 p$ and $19 q$ was observed. No K27 mutation subgroup of pediatric high-grade glioma was identified, despite the fact that four patients had symmetrical bi-thalamic gliomas, which are typically associated with H3K27M mutation (29). As expected, molecular differences were seen between pediatric and adult GC; the IDH subgroup was less common and no oligodendroglioma or RTK II subgroup was observed in children $(1,13,31)$.

\section{TREATMENT}

There is no standard treatment for patients with GC. While a long indolent course and prolonged survival are rarely observed (32), the disease more typically progresses rapidly, with a median survival of $<1$ year in patients not receiving antitumor therapy $(4,5)$.

\section{SURGERY AND TISSUE ACQUISITION}

All patients in whom GC is radiographically suspected should have a histopathologic confirmation. Given the diffuse involvement of a large brain volume, the role of surgery primarily lies in securing a tissue diagnosis. Some patients undergo partial resection of an area of T2-signal abnormality or T1 contrastenhancement to secure sufficient amount of tissue to overcome sampling error. When patients are symptomatic due to edema and mass effect, partial resection can be done with an aim of tumor debulking. It is unclear if extent of surgical resection 
provides any survival benefit. Perkins et al. reported outcomes in 30 GC patients of which 19 received biopsy and 11 had a partial resection (33). The median survival (21 versus 18 months, $p$ value $=0.96)$ did not reach statistical significance .

\section{RADIATION THERAPY}

The use of radiation therapy in GC is challenging due to the largefield involved and the apparent radioresistance of GC. Anecdotal evidence suggests stabilization of disease and resolution of neurological symptoms for a period of time in patients treated with radiation therapy alone (34-36). As GC histopathologically mirrors other gliomas, many institutions treat adult patients as high-grade glioma, with upfront radiation or chemo-radiation therapy. This approach raises concern in children given the large tumor volumes involved, the absence of a standard of care for children with high-grade gliomas, and the disputed evidence of efficacy of chemoradiation in pediatric malignant glioma (37). It is unclear whether radiation volume and/or dose correlates with outcome. Radiation therapy protocols have delivered radiation to involved field only, whole brain, or whole brain with a cone done to the involved field $(11,34)$. Whole brain radiotherapy doses ranging from 20 to $59 \mathrm{~Gy}(6,7,33,34)$ and regional radiotherapy doses from 54 to $66 \mathrm{GY}$ have been administered $(6,33)$. Chen et al. utilized a median radiation dose of $54.90 \mathrm{~Gy}$ and did not find any correlation between the total dose of radiation and survival (6). Four retrospective studies have reported a clinical response in $58 \%$ of patients and a radiographic response in $31 \%$ of patients $(7,33,34,38)$. Taillibert et al. reviewed a historic cohort of 296 patients and found that overall survival (OS) curves did not differ according to radiation treatment $(p=0.3)$ (5). In contrast, Chen et al. found the OS was significantly different $(p<0.01)$ in patients treated with (27.5 months) or without (6.5 months) radiation therapy (6). Despite clinical and radiographic improvement in many cases, response to radiation therapy is not durable and the evidence for its impact on OS is, at best, ambivalent.

\section{CHEMOTHERAPY}

Patients with GC usually receive chemotherapy alone or in conjunction with radiation. However, no study has demonstrated significant efficacy of chemotherapy in this disease. Table 1 reviews chemotherapy data derived from historical cohorts in patients with GC. NOA-05 is the only prospective clinical trial published to analyze the efficacy of primary chemotherapy in GC (39). This study was a phase II single arm study in which 35 patients with GC were treated with procarbazine and lomustine as upfront therapy. The median progression-free survival (PFS) was 14 months and median OS was 30 months. Although it is difficult to draw conclusions about superiority of upfront radiation versus chemotherapy regimen when comparing results of

TABLE 1 | Clinical studies reviewing treatment of patients with gliomatosis cerebri.

\begin{tabular}{|c|c|c|c|c|c|c|c|}
\hline Reference & Study type & Treatment & $\begin{array}{c}\text { No. of } \\
\text { patients }\end{array}$ & $\begin{array}{l}\text { Median PFS } \\
\text { (months) }\end{array}$ & $\begin{array}{l}\text { Median OS } \\
\text { (months) }\end{array}$ & Histology (\%) & Comments \\
\hline $\begin{array}{l}\text { Glas et al. (39), } \\
\text { NOA-05 }\end{array}$ & Prospective & PC & 35 & 14 & 30 & $\begin{array}{l}\text { DA } 20(57) \\
\text { AA } 7(20) \\
\text { OA } 2(6) \\
\text { AO } 2(6) \\
\text { GB } 4(11)\end{array}$ & $\begin{array}{l}\text { - MGMT in 12/25 (48\%) } \\
\text { - IDH1 mutation in 12/25 (48\%) } \\
\text { - PC in upfront setting }\end{array}$ \\
\hline Sanson et al. (4) & Retrospective & $\begin{array}{l}\text { PCV versus } \\
\text { TMZ }\end{array}$ & $\begin{array}{l}\text { 17-PCV } \\
\text { 46-TMZ }\end{array}$ & $\begin{array}{c}15.8 \\
16 \text { (no significant } \\
\text { difference) }\end{array}$ & $\begin{array}{c}25.6 \\
26.4 \text { (no significant } \\
\text { difference) }\end{array}$ & $\begin{array}{l}\text { OD } 43(72) \\
\text { OA } 6(10) \\
\text { A } 11(18)\end{array}$ & $\begin{array}{l}\text { - Both primary and secondary } \\
\text { GC with no focal mass }\end{array}$ \\
\hline $\begin{array}{l}\text { Soffietti et al. (41), } \\
\text { AINO }\end{array}$ & Retrospective & $\mathrm{TMZ}$ & 46 & 9 & 14 & $\begin{array}{l}\text { DA } 15(33) \\
\text { AA } 8(17) \\
\text { OD } 4(9) \\
\text { AO } 1(2) \\
\text { OA } 2(4) \\
\text { GB } 3(7) \\
\text { Others } 13(28)\end{array}$ & $\begin{array}{l}\text { - Both upfront and treatment at } \\
\text { progression }\end{array}$ \\
\hline Levin et al. (8) & Retrospective & $\mathrm{TMZ}$ & 11 & 13 & Not reached & $\begin{array}{l}\text { DA } 2(18) \\
\text { OD } 6(55) \\
\text { AO } 1(9) \\
\text { OA } 1(9) \\
\text { GB } 1(9)\end{array}$ & $\begin{array}{l}\text { - } 6 \text { patients received PCV upfront } \\
\text { - Patients with GC with or without } \\
\text { focal mass }\end{array}$ \\
\hline Kaloshi et al. (42) & Retrospective & TMZ & 25 & 18 & 37.7 & $\begin{array}{l}\text { Oligodendroglial } \\
14(56) \\
\text { Astrocytic } 9 \text { (36) } \\
\text { Others } 2(8)\end{array}$ & $\begin{array}{l}\text { - Primary and secondary GC } \\
\text { - Upfront treatment }\end{array}$ \\
\hline
\end{tabular}

$A A$, anaplastic astrocytoma; $A O$, anaplastic oligodendroglioma; $D A$, diffuse astrocytoma; GB, glioblastoma; $O A$, oligoastrocytoma; $O D$, oligodendroglioma; $P C$, procarbazine and Iomustine; TMZ, temozolomide. 
this study to retrospective historical cohorts who received radiotherapy only (median OS 11.4-38.4 months) (7, 33, 34, 38), the NOA-05 trial results suggest that initial treatment with procarbazine and lomustine may have potential clinical benefit for patients with GC.

Temozolomide is widely used for treatment of adult malignant gliomas and is often used in treatment of GC. Samson et al. retrospectively compared response rate to procarbazine, vincristine and lomustine (PCV) versus temozolomide in a series of 63 patients with GC. No significant difference was observed in the PCV and temozolomide groups in PFS (15.8 versus 16 months) or OS (25.6 versus 26.4 months), but increased toxicity was noted in the PCV group. Retrospective studies have demonstrated that temozolomide can be used in the treatment of GC, both as initial therapy and at progression with a median PFS and OS ranging from 9 to 18 and 14 to 37.7 months, respectively $(4,8,41,42)$. A report from Levin et al. suggested that temozolomide may also be used after initial tumor progression with PCV treatment (8). Of 2 patients whose treatment was changed from PCV to temozolomide, one had continued disease progression but the other was stable for 12 months. Given the variability in PFS and OS in historical cohorts of patients with GC, randomized phase II studies may better elucidate the roles of chemotherapy in this disease.

Patients with GC who have oligodendroglial pathology and $1 p / 19 q$ codeletions have a higher radiographic response rate, PFS, and OS when treated with temozolomide as compared to patients with non-oligodendroglial GC $(4,42)$. These data are not surprising given our current knowledge about the chemosensitive nature of oligodendroglial tumors and longer overall patient survival when compared with those with astrocytic tumors. Similar evidence of chemosensitivity can be found from some case reports and studies where nitrosourea-based regimens were used upfront $(4,39,43,44)$. From current literature, it appears that both temozolomide- and nitrosourea-based regimens may be useful for initial treatment of adult patients with oligodendroglial GC, yet no conclusion can be drawn about the superiority of one treatment over another.

As most cases of GC show a lack of contrast-enhancement on MRI and CT, neovascularization is considered to be rare or absent in this disease (45). In contrast to this assumption, a study found strong VEGF expression in five of six patients and COX2 expression in four of six patients despite the absence of contrast-enhancement on MRI. Additionally, histopathology and CD31 antibody studies demonstrated vascular proliferation in gliomatosis affected areas. Patients in this non-randomized study were treated with a combination of low-dose temozolomide and celecoxib and had PFS of 6-18 months (46).

While new treatment paradigms using immunotherapy are being developed for high-grade gliomas, these have not formally evaluated in patients with GC. Generally, tumor cells survive by dysregulating the body's immune checkpoints by overexpressing immunosuppressive surface ligands such as programmed cell death-1 (PD-1) and cytotoxic lymphocyte-associated protein-4 (CTLA-4). Immune checkpoint inhibitors such as nivolumab (anti-PD-1), pembrolizumab (anti-PD-1), and ipilimumab (anti-CTLA-4) are being investigated for CNS tumors, including glioblastoma. With their success in various solid tumors like melanoma and non-small cell lung cancer $(47,48)$, they may be of potential benefit in a heterogeneous disease entity such as GC and thus need to be investigated. Additionally, clinical trials (NCT02746081) are underway to test IDH inhibitors in gliomas $(49,50)$ as the IDH mutation can be found in $17-48 \%$ of adults with GC $(1,13,39)$. Little is known about metabolism and metabolic defects in GC. However, a major issue in evaluating efficacy of chemotherapeutic agents for GC patients is inconsistent inclusion in clinical trials, lack of GC-specific cohorts, and variable definitions of GC for eligibility.

\section{CHALLENGES}

The initial challenge in management of GC is timely diagnosis. Symptoms and MRI findings are non-specific, therefore a confirmed diagnosis of GC is delayed. When patients present with advanced symptoms and large tumor volumes, treatment options can be limited, although whether earlier diagnosis and treatment is associated with better outcomes is unknown. Because of the relatively extensive differential diagnosis, tissue confirmation is critical. However, biopsy results in only limited tissue available for diagnosis and molecular testing. Advanced molecular studies are necessary to further our understanding of GC and identify potential targets for therapy. Understanding the tumor pathophysiology and specifically the biology behind the extreme invasiveness of tumor cells is a first step toward developing novel therapeutics for GC. In the First International Gliomatosis cerebri Group Meeting held in Paris, France in March 2015, it was suggested that at least two different biopsy samples using the same needle tract at different depth be obtained to increase tissue sampling (16). Additionally, advanced imaging modalities like MRS, perfusion MR and FDG-PET along with standard MRI, should be incorporated in GC management as they can aid in delineating the extent of disease, selecting the appropriate surgical site for biopsy and in treatment assessment.

Given the lack of randomized controlled trials, it is unclear if radiation therapy or chemotherapy benefits patients with GC, if GC subtypes have different responses, or if pediatric and adult GC differ biologically. Only one prospective study, NOA-05, has been published to date and this was a non-randomized study using a historical cohort. Treatment conclusions gleaned from retrospective series are subject to publication bias and offer limited information due to small sample size. Randomized studies for radiation therapy are complicated by the heterogeneity of the disease. Additionally, large-field partial brain radiotherapy or whole brain radiotherapy may have a significant impact on quality of life.

Because of GC's clinical, molecular and pathological heterogeneity, it is difficult to evaluate efficacy of a specific therapy as the study population and historical cohorts vary. Defining criteria for randomization is critical. Several studies have attempted to prognosticate survival and outcomes in retrospective analysis using clinical, radiographic and, more recently, molecular data. With our current knowledge that the molecular profiles of adult and pediatric GC are not distinct from glial tumors of corresponding WHO grades, the next pertinent question is whether grade and histology of the tumor plays any role at all in prognosis and prediction of outcome. While two studies with a relatively large 
sample size retrospectively analyzed the relationship of tumor grade and survival $(5,6)$, others did not find that tumor grade was prognostic of outcome $(1,4,13,39,51)$. Recent data from genome-wide DNA methylation analysis on 25 patients have also shown that WHO grades were not prognostic of outcomes, but patients with a molecular classification of classic/RTK2 or mesenchymal glioblastoma fared worse (1). Sanson et al. showed that a pure oligodendroglial pathology of GC was associated with a significantly better outcome (4), but this was not validated by Glas et al., likely as his study included only four patients with oligodendroglial pathology, all of which were oligoastrocytomas (39). Various studies have reported an OS of 18-35 months for grade II, 12-29 months for grade III and 9-36 months for grade IV GC $(1,4,5,34,51)$. Even though these data are highly variable, contrast this to OS of 8-13 years in grade II gliomas (52), 37 months to 15 years for grade III gliomas $(53,54)$, and $15-16$ months in glioblastoma $(55,56)$. It is quite clear that patients with GC fare much worse than patients with diffuse gliomas of corresponding grades.

Knowledge about molecular profile has helped to refine prognostication in other adult diffuse gliomas. Mutations affecting the IDH genes 1 and 2 are associated with longer OS as compared to patients with IDH wild type genes, although this does not hold true for pediatric patients. The IDH mutation can be found in up to $48 \%$ of adult patients with GC $(1,40,57)$, but there is no clear relationship with outcome $(p=0.08)(1)$ or to better prognosis $(39,40,57,58)$. Molecular data and its relationship with survival are scanty in children. To date, of 19 pediatric patients tested for IDH mutations in two different studies, only $3(16 \%)$ patients had tumors with an IDH mutation $(13,31)$. MGMT promoter methylation is another molecular marker related to better outcomes in adults with malignant glioma $(1,39)$, but its applicability in children is unclear (13).

A number of studies looked at the association of age and outcome to determine if older age in adults is related to a poor outcome, but no convincing evidence is seen $(4-6,39,51)$. In a small cohort of pediatric patients, Armstrong et al. found that age at diagnosis was a significant predictor of OS with children diagnosed in the first decade of life faring poorly (11), however, another pediatric study found no such relationship (13). Similarly, the relationship between good performance status and outcome is not consistent with studies reporting a better outcome with a high performance status $(5,6)$, while others failed to find an association $(4,39)$. No correlation is found with presenting neurological symptoms and outcome $(6,13)$. Most studies have found no relationship with the MRI appearance of lesion (contrast-enhancement, symmetrical presentation) with patient prognosis $(1,6,11,13,39)$. However, children who present with symmetrical bi-thalamic involvement show a poor prognosis (13). Two studies have found that patients with substantial gray matter involvement (e.g., thickening of cortex, insula, basal ganglia and thalamus) have worse outcomes than patients with predominantly white matter involvement (diffuse swelling of hemisphere, swelling of corpus callosum, and loss of gray-white differentiation) $(59,60)$. The conclusions from these studies are limited by the relatively small numbers of patients.

\section{CONCLUSION}

Gliomatosis cerebri remains a poorly understood entity that affects all age groups. Despite aggressive treatment, patients have a uniformly poor outcome. There are a paucity of studies evaluating the biology and pathophysiology of this disease. Although no longer considered a distinct pathologic entity, GC represents a disease with a unique phenotype. We now have the tools to increase our knowledge of the molecular biology of GC; further study of the biology driving this migratory and invasive growth pattern of tumor cells and its microenvironment is necessary to better define GC. These investigations may lead to new therapeutic targets or more rational therapeutic management of these patients.

\section{AUTHOR CONTRIBUTIONS}

Both authors jointly developed the design and arguments for the paper, drafted the manuscript, reviewed, approved the final manuscript, and are accountable for all aspects of the work.

\section{ACKNOWLEDGMENT}

This project has been fully funded by the Intramural Research Program of the National Institutes of Health.

\section{REFERENCES}

1. Herrlinger U, Jones DT, Glas M, Hattingen E, Gramatzki D, Stuplich M, et al. Gliomatosis cerebri: no evidence for a separate brain tumor entity. Acta Neuropathol (2015) 131(2):309-19. doi:10.1007/s00401-015-1495-z

2. Jennings MT, Frenchman M, Shehab T, Johnson MD, Creasy J, LaPorte K, et al. Gliomatosis cerebri presenting as intractable epilepsy during early childhood. JChild Neurol (1995) 10(1):37-45. doi:10.1177/088307389501000111

3. Louis DN, Perry A, Reifenberger G, von Deimling A, Figarella-Branger D, Cavenee WK, et al. The 2016 World Health Organization Classification of Tumors of the Central Nervous System: a summary. Acta Neuropathol (2016) 131(6):803-20. doi:10.1007/s00401-016-1545-1

4. Sanson M, Cartalat-Carel S, Taillibert S, Napolitano M, Djafari L, Cougnard J, et al. Initial chemotherapy in gliomatosis cerebri. Neurology (2004) 63(2):270-5. doi:10.1212/01.WNL.0000129985.39973.E4

5. Taillibert S, Chodkiewicz C, Laigle-Donadey F, Napolitano M, CartalatCarel S, Sanson M. Gliomatosis cerebri: a review of 296 cases from the ANOCEF database and the literature. J Neurooncol (2006) 76(2):201-5. doi:10.1007/s11060-005-5263-0

6. Chen S, Tanaka S, Giannini C, Morris J, Yan ES, Buckner J, et al. Gliomatosis cerebri: clinical characteristics, management, and outcomes. J Neurooncol (2013) 112(2):267-75. doi:10.1007/s11060-013-1058-x

7. Elshaikh MA, Stevens GH, Peereboom DM, Cohen BH, Prayson RA, Lee SY, et al. Gliomatosis cerebri. Cancer (2002) 95(9):2027-31. doi:10.1002/ cncr.10909

8. Levin N, Gomori JM, Siegal T. Chemotherapy as initial treatment in gliomatosis cerebri: results with temozolomide. Neurology (2004) 63(2):354-6. doi:10.1212/01.WNL.0000130249.41341.58

9. Rajz GG, Nass D, Talianski E, Pfeffer R, Spiegelmann R, Cohen ZR. Presentation patterns and outcome of gliomatosis cerebri. Oncol Lett (2012) 3(1): 209-13. doi:10.3892/ol.2011.445 
10. George E, Settler A, Connors S, Greenfield JP. Pediatric gliomatosis cerebri: a review of 15 years. J Child Neurol (2015) 31(3):378-87. doi:10.1177/ 0883073815596612

11. Armstrong GT, Phillips PC, Rorke-Adams LB, Judkins AR, Localio AR, Fisher MJ. Gliomatosis cerebri: 20 years of experience at the Children's Hospital of Philadelphia. Cancer (2006) 107(7):1597-606. doi:10.1002/cncr. 22210

12. Rudà R, Bertero L, Sanson M. Gliomatosis cerebri: a review. Curr Treat Options Neurol (2014) 16(2):1-9. doi:10.1007/s11940-013-0273-2

13. Broniscer A, Chamdine O, Hwang S, Lin T, Pounds S, Onar-Thomas A, et al. Gliomatosis cerebri in children shares molecular characteristics with other pediatric gliomas. Acta Neuropathol (2016) 131(2):299-307. doi: 10.1007/s00401-015-1532-y

14. Shin YM, Chang KH, Han MH, Myung NH, Chi JG, Cha SH, et al. Gliomatosis cerebri: comparison of MR and CT features. AJNR Am J Roentgenol (1993) 161(4):859-62. doi:10.2214/ajr.161.4.8372774

15. Bendszus M, Warmuth-Metz M, Klein R, Burger R, Schichor C, Tonn JC, et al. MR spectroscopy in gliomatosis cerebri. AJNR Am J Neuroradiol (2000) 21(2):375-80.

16. Greenfield JP, Castaneda Heredia A, George E, Kieran MW, Morales La Madrid A. Gliomatosis cerebri: a consensus summary report from the First International Gliomatosis cerebri Group Meeting, March 26-27, 2015, Paris, France. Pediatr Blood Cancer (2016) 63(12):2072-7. doi:10.1002/pbc.26169

17. Singhal N, Agarwal V. Pediatric gliomatosis cerebri mimicking tubercular encephalitis. JPediatr Neurosci (2015) 10(4):379-81. doi:10.4103/ 1817-1745.174430

18. Buis DR, van der Valk P, De Witt Hamer PC. Subcutaneous tumor seeding after biopsy in gliomatosis cerebri. J Neurooncol (2012) 106:431-5. doi:10.1007/ s11060-011-0678-2

19. Kararizou E, Likomanos D, Gkiatas K, Markou I, Triantafyllou N, Kararizos G. Magnetic resonance spectroscopy: a noninvasive diagnosis of gliomatosis cerebri. Magn Reson Imaging (2006) 24(2):205-7. doi:10.1016/j. mri.2005.10.032

20. Krouwer HG, Kim TA, Rand SD, Prost RW, Haughton VM, Ho KC, et al. Single-voxel proton MR spectroscopy of nonneoplastic brain lesions suggestive of a neoplasm. AJNR Am J Neuroradiol (1998) 19(9):1695-703.

21. Chang L, Ernst T, Tornatore C, Aronow H, Melchor R, Walot I, et al. Metabolite abnormalities in progressive multifocal leukoencephalopathy by proton magnetic resonance spectroscopy. Neurology (1997) 48(4):836-45. doi:10.1212/WNL.48.4.836

22. Yang S, Wetzel S, Law M, Zagzag D, Cha S. Dynamic contrast-enhanced T2*-weighted MR imaging of gliomatosis cerebri. AJNR Am J Neuroradiol (2002) 23(3):350-5.

23. Dexter MA, Parker GD, Besser M, Ell J, Fulham MJ. MR and positron emission tomography with fludeoxyglucose F 18 in gliomatosis cerebri. AJNR Am J Neuroradiol (1995) 16(7):1507-10.

24. Plowman PN, Saunders CA, Maisey MN. Gliomatosis cerebri: disconnection of the cortical grey matter, demonstrated on PET scan. Br J Neurosurg (1998) 12(3):240-4. doi:10.1080/02688699845069

25. Fuller GN, Kros JM. Gliomatosis cerebri. 4th ed. In: Louis DN, Ohgaki H, Wiestler OD, Cavenee WK, editors. WHO Classification of Tumours of the Central Nervous System. Lyon, France: International Agency for Research on Cancer (2007). p. 50-2.

26. Kandler RH, Smith CM, Broome JC, Davies-Jones GA. Gliomatosis cerebri: a clinical, radiological and pathological report of four cases. $\mathrm{Br}$ J Neurosurg (1991) 5(2):187-93. doi:10.3109/02688699108998466

27. Hovestadt V, Remke M, Kool M, Pietsch T, Northcott PA, Fischer R, et al. Robust molecular subgrouping and copy-number profiling of medulloblastoma from small amounts of archival tumour material using high-density DNA methylation arrays. Acta Neuropathol (2013) 125(6):913-6. doi:10.1007/ s00401-013-1126-5

28. Sturm D, Witt H, Hovestadt V, Khuong-Quang DA, Jones DT, Konermann C, et al. Hotspot mutations in H3F3A and IDH1 define distinct epigenetic and biological subgroups of glioblastoma. Cancer Cell (2012) 22(4):425-37. doi:10.1016/j.ccr.2012.08.024

29. Korshunov A, Ryzhova M, Hovestadt V, Bender S, Sturm D, Capper D, et al. Integrated analysis of pediatric glioblastoma reveals a subset of biologically favorable tumors with associated molecular prognostic markers. Acta Neuropathol (2015) 129(5):669-78. doi:10.1007/s00401-015-1405-4
30. Pajtler KW, Witt H, Sill M, Jones DT, Hovestadt V, Kratochwil F, et al. Molecular classification of ependymal tumors across all CNS compartments, histopathological grades, and age groups. Cancer Cell (2015) 27(5):728-43. doi:10.1016/j.ccell.2015.04.002

31. Seiz M, Tuettenberg J, Meyer J, Essig M, Schmieder K, Mawrin C, et al. Detection of IDH1 mutations in gliomatosis cerebri, but only in tumors with additional solid component: evidence for molecular subtypes. Acta Neuropathol (2010) 120(2):261-7. doi:10.1007/s00401-010-0701-2

32. Cervos-Navarro J, Artigas J, Aruffo C, Iglesias J. The fine structure of gliomatosis cerebri. Virchows Arch A Pathol Anat Histopathol (1987) 411(1):93-8. doi:10.1007/BF00734520

33. Perkins GH, Schomer DF, Fuller GN, Allen PK, Maor MH. Gliomatosis cerebri: improved outcome with radiotherapy. Int J Radiat Oncol Biol Phys (2003) 56(4):1137-46. doi:10.1016/S0360-3016(03)00293-1

34. Cozad SC, Townsend P, Morantz RA, Jenny AB, Kepes JJ, Smalley SR. Gliomatosis cerebri. Results with radiation therapy. Cancer (1996) 78(8):1789-93. doi:10.1002/(SICI)1097-0142(19961015)78:8<1789::AIDCNCR20>3.0.CO;2-T

35. Artigas J, Cervos-Navarro J, Iglesias JR, Ebhardt G. Gliomatosis cerebri: clinical and histological findings. Clin Neuropathol (1985) 4(4):135-48.

36. Yanaka K, Kamezaki T, Kobayashi E, Matsueda K, Yoshii Y, Nose T. MR imaging of diffuse glioma. AJNR Am J Neuroradiol (1992) 13(1): 349-51.

37. Fangusaro J, Warren KE. Unclear standard of care for pediatric high grade glioma patients. J Neurooncol (2013) 113(2):341-2. doi:10.1007/s11060013-1104-8

38. Kim DG, Yang HJ, Park IA, Chi JG, Jung HW, Han DH, et al. Gliomatosis cerebri: clinical features, treatment, and prognosis. Acta Neurochir (1998) 140(8):755-62. doi:10.1007/s007010050176

39. Glas M, Bahr O, Felsberg J, Rasch K, Wiewrodt D, Schabet M, et al. NOA-05 phase 2 trial of procarbazine and lomustine therapy in gliomatosis cerebri. Ann Neurol (2011) 70(3):445-53. doi:10.1002/ana.22478

40. Glas M, Rasch K, Wiewrodt D, Weller M, Herrlinger U. Procarbazine and CCNU as initial treatment in gliomatosis cerebri. Oncology (2008) 75(3-4):182-5. doi:10.1159/000163057

41. Soffietti R, Rudà R, Laguzzi E, Buttolo L, Pace A, Carapella C, et al., editors. Treatment of gliomatosis cerebri with temozolomide: a retrospective study of the AINO. ASCO Annual Meeting Proceedings. Chicago: Italian Association for Neuro-Oncology (2007).

42. Kaloshi G, Everhard S, Laigle-Donadey F, Marie Y, Navarro S, Mokhtari K, et al. Genetic markers predictive of chemosensitivity and outcome in gliomatosis cerebri. Neurology (2008) 70(8):590-5. doi:10.1212/01.wnl. 0000299896.65604.ae

43. Fukushima Y, Nakagawa H, Tamura M. Combined surgery, radiation, and chemotherapy for oligodendroglial gliomatosis cerebri. Br J Neurosurg (2004) 18(3):306-10. doi:10.1080/02688690410001732823

44. Louis E, Keime-Guibert F, Delattre JY, Sanson M. Dramatic response to chemotherapy in oligodendroglial gliomatosis cerebri. Neurology (2003) 60(1):151. doi:10.1212/WNL.60.1.151

45. Bernsen H, Van der Laak J, Kusters B, Van der Ven A, Wesseling P. Gliomatosis cerebri: quantitative proof of vessel recruitment by cooptation instead of angiogenesis. J Neurosurg (2005) 103(4):702-6. doi:10.3171/jns.2 005.103.4.0702

46. Seiz M, Kohlhof P, Brockmann MA, Neumaier-Probst E, Hermes P, Vond A, et al. First experiences with low-dose anti-angiogenic treatment in gliomatosis cerebri with signs of angiogenic activity. Anticancer Res (2009) 29(8):3261-7.

47. Hodi FS, O’Day SJ, McDermott DF, Weber RW, Sosman JA, Haanen JB, et al. Improved survival with ipilimumab in patients with metastatic melanoma. N Engl J Med (2010) 363(8):711-23. doi:10.1056/NEJMoa1003466

48. Socinski MA. Advances in immuno-oncology: immune checkpoint inhibitors in non-small cell lung cancer-introduction. Semin Oncol (2015) 42(Suppl 2):S1-2. doi:10.1053/j.seminoncol.2015.09.018

49. Rohle D, Popovici-Muller J, Palaskas N, Turcan S, Grommes C, Campos C, et al. An inhibitor of mutant IDH1 delays growth and promotes differentiation of glioma cells. Science (2013) 340(6132):626-30. doi:10.1126/science. 1236062

50. Popovici-Muller J, Saunders JO, Salituro FG, Travins JM, Yan S, Zhao F, et al. Discovery of the first potent inhibitors of mutant IDH1 that lower 
tumor 2-HG in vivo. ACS Med Chem Lett (2012) 3(10):850-5. doi:10.1021/ $\mathrm{ml} 300225 \mathrm{~h}$

51. Ware ML, Hirose Y, Scheithauer BW, Yeh RF, Mayo MC, Smith JS, et al. Genetic aberrations in gliomatosis cerebri. Neurosurgery (2007) 60(1):150-8; discussion 8. doi:10.1227/01.NEU.0000249203.73849.5D

52. Buckner JC, Shaw EG, Pugh SL, Chakravarti A, Gilbert MR, Barger GR, et al. Radiation plus procarbazine, CCNU, and vincristine in low-grade glioma. N Engl J Med (2016) 374(14):1344-55. doi:10.1056/NEJMoa1500925

53. Strowd RE, Abuali I, Ye X, Lu Y, Grossman SA. The role of temozolomide in the management of patients with newly diagnosed anaplastic astrocytoma: a comparison of survival in the era prior to and following the availability of temozolomide. J Neurooncol (2016) 127(1):165-71. doi:10.1007/s11060015-2028-2

54. Cairncross G, Wang M, Shaw E, Jenkins R, Brachman D, Buckner J, et al. Phase III trial of chemoradiotherapy for anaplastic oligodendroglioma: long-term results of RTOG 9402. J Clin Oncol (2013) 31(3):337-43. doi:10.1200/JCO.2012.43.2674

55. Gilbert MR, Dignam JJ, Armstrong TS, Wefel JS, Blumenthal DT, Vogelbaum MA, et al. A randomized trial of bevacizumab for newly diagnosed glioblastoma. N Engl J Med (2014) 370(8):699-708. doi:10.1056/ NEJMoa1308573

56. Stupp R, Mason WP, van den Bent MJ, Weller M, Fisher B, Taphoorn MJ, et al. Radiotherapy plus concomitant and adjuvant temozolomide for glioblastoma. N Engl J Med (2005) 352(10):987-96. doi:10.1056/ NEJMoa043330
57. Kwon MJ, Kim ST, Kwon MJ, Kong DS, Lee D, Park S, et al. Mutated IDH1 is a favorable prognostic factor for type 2 gliomatosis cerebri. Brain Pathol (2012) 22(3):307-17. doi:10.1111/j.1750-3639.2011.00532.x

58. Desestret V, Ciccarino P, Ducray F, Criniere E, Boisselier B, Labussiere M, et al. Prognostic stratification of gliomatosis cerebri by IDH1 R132H and INA expression. J Neurooncol (2011) 105(2):219-24. doi:10.1007/s11060-011-0587-4

59. Kaloshi G, Guillevin R, Martin-Duverneuil N, Laigle-Donadey F, Psimaras D, Marie Y, et al. Gray matter involvement predicts chemosensitivity and prognosis in gliomatosis cerebri. Neurology (2009) 73(6):445-9. doi:10.1212/WNL.0b013e3181b163e2

60. Chappé C, Riffaud L, Tréguier C, Carsin-Nicol B, Veillard D, Chiforeanu DC, et al. Primary gliomatosis cerebri involving gray matter in pediatrics: a distinct entity? A multicenter study of 14 cases. Childs Nerv Syst (2013) 29(4):565-71. doi:10.1007/s00381-012-2016-1

Conflict of Interest Statement: The authors declare that the research was conducted in the absence of any commercial or financial relationships that could be construed as a potential conflict of interest.

Copyright $\odot 2017$ Ranjan and Warren. This is an open-access article distributed under the terms of the Creative Commons Attribution License (CC BY). The use, distribution or reproduction in other forums is permitted, provided the original author(s) or licensor are credited and that the original publication in this journal is cited, in accordance with accepted academic practice. No use, distribution or reproduction is permitted which does not comply with these terms. 